\title{
FACTORIZATION BY INVARIANT EMBEDDING OF ELLIPTIC PROBLEMS IN A CIRCULAR DOMAIN
}

\author{
J. Henry \\ INRIA-Futurs \\ $M A B$ Université Bordeaux 1 \\ 351, cours de la libération, 33405 Talence, France \\ jacques.henry@inria.fr
}

B. Louro

Departamento de Matemática, Faculdade de Ciências e Tecnologia

Universidade Nova de Lisboa, 2829-516 Caparica, Portugal

bjl@ft.unl.pt

\author{
M.C. Soares* \\ Departamento de Matemática, Faculdade de Ciências e Tecnologia \\ Universidade Nova de Lisboa, 2829-516 Caparica, Portugal \\ mcs@fct.uni.pt
}

\begin{abstract}
We present a method to factorize a second order elliptic boundary value problem in a circular domain, in a system of uncoupled first order initial value problems. We use a space invariant embedding technique along the radius of the circle, in both an increasing and a decreasing way. This technique is inspired in the temporal invariant embedding used by J.-L. Lions for the control of parabolic systems. The singularity at the origin for the initial value problems is studied.
\end{abstract}

Keywords: Factorization, Riccati equation, invariant embedding.

\section{Introduction}

The technique of invariant embedding was first introduced by Bellman ([2]) and was formally used by Angel and Bellman ([1]) in the resolution 
of Poisson's problem defined over a rectangle. J.L. Lions ([5]) gave a justification for this invariant embedding in the computation of the optimal feedback in the framework of Optimal Control of evolution equations of parabolic type. Henry and Ramos ([3]) presented a justification for the invariant embedding of Poisson's problem in a cylindrical domain. The problem is embedded in a family of similar problems defined on subcylinders limited by a moving boundary. They obtained a factorization in two uncoupled problems of parabolic type, in opposite directions. In this paper, we want to generalize this method to other types of geometries and, in particular, to the case where the family of surfaces which limits the sub-domains, starts on the outside boundary of the domain and shrinks to a point. We present here the simple situation where $\Omega$ $\left(\operatorname{resp} \Omega_{s}\right.$ ) is a disk of $\mathbb{R}^{2}$ with radius $a$ (resp $s$ ) and centered on the origin and where the sub-domains defined by the invariant embedding are both the annuli $\Omega \backslash \Omega_{s}, s \in(0, a)$ ([4]) and the family of disks $\Omega_{s}$, $s \in(0, a)$. This factorization can be viewed as an infinite dimensional extension of the block Gauss factorization for linear systems.

\section{Motivation}

Given $f \in L^{2}(0,1), y_{0}, y_{1} \in \mathbb{R}, q \in \mathbb{R}^{+}, p \in \mathbb{R}^{+} \backslash\{0\}$, let $y$ be the solution of the following boundary value problem:

$$
\left\{\begin{array}{l}
\left.-p \frac{d^{2} y}{d x^{2}}+q y=f, x \in\right] 0,1[ \\
\frac{d y}{d x}(0)=y_{0} \\
y(1)=y_{1}
\end{array}\right.
$$

Considering the operator $A=-p \frac{d^{2}}{d x^{2}}+q$, the natural way to factorize it, is by searching $\alpha, \beta$ such that $A \stackrel{d x^{2}}{=}-p\left(\frac{d}{d x}+\beta(x)\right)\left(\frac{d}{d x}-\alpha(x)\right)$.

Then, for each $\varphi \in \mathcal{C}^{2}((0,1))$ we have

$$
A(\varphi)=-p \frac{d^{2} \varphi}{d x^{2}}+p(\alpha-\beta) \frac{d \varphi}{d x}+p\left(\frac{d \alpha}{d x}+\alpha \beta\right) \varphi
$$

Thus, we must have $\alpha=\beta$ and $\frac{d \beta}{d x}+\beta^{2}=\frac{q}{p}$. If we set $\beta(0)=0$ and $\xi=-\frac{d y}{d x}+\beta y$, we find $\xi(0)=-y_{0}$ and the following system of uncoupled equations: 


$$
\begin{cases}\frac{d \beta}{d x}+\beta^{2}=\frac{q}{p}, & \beta(0)=0 \\ \frac{d \xi}{d x}+\beta \xi=\frac{f}{p}, & \xi(0)=-y_{0} \\ \frac{d y}{d x}-\beta y=-\xi, & y(1)=y_{1}\end{cases}
$$

We point out that the equation in $\beta$ is a Riccati equation.

\section{Formulation of the Problem and a Regularization Result}

We consider the Dirichlet problem for the Poisson equation defined over $\Omega$.

$$
(\mathcal{P}) \quad-\Delta u=f, \text { in } \Omega ;\left.u\right|_{\Gamma_{a}}=u_{0},
$$

where $\Gamma_{s}$ denotes the circle of radius $s$ and center at the origin, $f \in L^{2}(\Omega)$ and $u_{0} \in H^{1 / 2}\left(\Gamma_{a}\right)$. We assume the additional regularity around the origin $f \in \mathcal{C}^{0, \alpha}(\mathcal{O}), \mathcal{O}$ being a neighborhood of the origin. Introducing polar coordinates, $\hat{u}(\rho, \theta)=u\left(x_{1}, x_{2}\right)$ satisfies

$$
(\hat{\mathcal{P}})\left\{\begin{array}{l}
\left.-\frac{1}{\rho} \frac{\partial}{\partial \rho}\left(\rho \frac{\partial \hat{u}}{\partial \rho}\right)-\frac{1}{\rho^{2}} \frac{\partial^{2} \hat{u}}{\partial \theta^{2}}=\hat{f}, \text { in }\right] 0, a[\times \mathcal{I} \\
\left.\hat{u}\right|_{\Gamma_{a}}=\hat{u}_{0} ; \hat{u} 2 \pi \text { - periodic with respect to } \theta
\end{array}\right.
$$

where $\mathcal{I}=] 0,2 \pi[$. However, by doing this, we introduce a singularity at the origin. Furthermore the analogous of the computation done in [3] would need to know $u(0)$ which is not a data of the problem.

In order to avoid this difficulty we start by defining the following intermediate problem:

$$
\left(\mathcal{P}_{\varepsilon}\right)\left\{\begin{array}{l}
-\Delta u_{\varepsilon}=f, \text { in } \Omega \backslash \Omega_{\varepsilon} ;\left.u_{\varepsilon}\right|_{\Gamma_{a}}=u_{0} \\
\int_{\Gamma_{\varepsilon}} \frac{\partial u_{\varepsilon}}{\partial n} \mathrm{~d} \Gamma=0 ;\left.u_{\varepsilon}\right|_{\Gamma_{\varepsilon}} \text { is constant }
\end{array}\right.
$$

where $\Omega_{\varepsilon}$ is a circular domain of radius $0<\varepsilon<a$ and concentric with $\Omega$. It's easy to see that this problem is well posed.

THeOREM 1 When $\varepsilon \rightarrow 0, \tilde{u}_{\varepsilon}$, defined as $\tilde{u}_{\varepsilon}=\left\{\begin{array}{l}u_{\varepsilon}, \text { in } \Omega \backslash \Omega_{\varepsilon} \\ u_{\varepsilon}=u_{\varepsilon} \mid \Gamma_{\varepsilon}, \text { in } \Omega_{\varepsilon}\end{array}\right.$, where $u_{\varepsilon}$ is the solution of problem $\left(\mathcal{P}_{\varepsilon}\right)$ converges to $u$, solution of problem $(\mathcal{P})$, in $H^{1}(\Omega)$.

We can write problem $\left(\mathcal{P}_{\varepsilon}\right)$ in polar coordinates restricting problem $(\hat{\mathcal{P}})$ over $] \varepsilon, a\left[\times \mathcal{I}\right.$ and joining the boundary conditions $\left.\hat{u}_{\varepsilon}\right|_{\Gamma_{\varepsilon}}$ constant, $\int_{\Gamma_{\varepsilon}} \frac{\partial \hat{u}_{\varepsilon}}{\partial \rho} \mathrm{d} \theta=0$ 


\section{Factorization by Invariant Embedding}

We embed problem $\left(\mathcal{P}_{\varepsilon}\right)$ in a family of similar problems $\left(\mathcal{P}_{s, h}\right)$ defined on the annulus $\left.\Omega \backslash \Omega_{s}, s \in\right] \varepsilon, a$ [ and satisfying an additional Neumann boundary condition in $\Gamma_{s}$; in terms of polar coordinates we find

$$
\left(\hat{\mathcal{P}}_{s, h}\right)\left\{\begin{array}{l}
\left.-\frac{1}{\rho} \frac{\partial}{\partial \rho}\left(\rho \frac{\partial \hat{u}_{s}}{\partial \rho}\right)-\frac{1}{\rho^{2}} \frac{\partial^{2} \hat{u}_{s}}{\partial \theta^{2}}=\hat{f}, \text { in }\right] s, a[\times \mathcal{I} \\
\hat{u}_{s} \mid \Gamma_{a}=\hat{u}_{0}, \hat{u}_{s} 2 \pi-\text { periodic with respect to } \theta \\
\left.\frac{\partial \hat{u}_{s}}{\partial \rho}\right|_{\Gamma_{s}}=h
\end{array}\right.
$$

Since $\left.\frac{\partial \hat{u}_{\varepsilon}}{\partial \rho}\right|_{\Gamma_{\varepsilon}}$ is well determined through the conditions " $\hat{u}_{\varepsilon_{\Gamma_{\varepsilon}}}$ constant" and " $\int_{\Gamma_{\varepsilon}} \frac{\partial \hat{u}_{\varepsilon}}{\partial \rho} \mathrm{d} \theta=0$ ", it's clear that $\left(\hat{\mathcal{P}}_{\varepsilon}\right)$ belongs to the family $\left(\hat{\mathcal{P}}_{s, h}\right)$ for $s=\varepsilon$.

Defining $H_{\rho, P}^{1}(\mathcal{I})$ as the space of periodic functions $v$ of $\theta$, verifying $v \in L^{2}(\mathcal{I})$ and $\frac{1}{\rho} \frac{\partial v}{\partial \theta} \in L^{2}(\mathcal{I})$, we take $h \in H_{\rho, P}^{1 / 2}(\mathcal{I})^{\prime}$, where $H_{\rho, P}^{1 / 2}(\mathcal{I})=$ $\left[H_{\rho, P}^{1}(\mathcal{I}), L^{2}(\mathcal{I})\right]_{1 / 2}$.

For every $s \in[\varepsilon, a), h \in H_{\rho, P}^{1 / 2}(\mathcal{I})^{\prime}$ we define $P(s) h=\gamma_{s_{\Gamma_{s}}}$, where $\gamma_{s}$ is the solution of

$$
\left\{\begin{array}{l}
\left.-\frac{1}{\rho} \frac{\partial}{\partial \rho}\left(\rho \frac{\partial \gamma_{s}}{\partial \rho}\right)-\frac{1}{\rho^{2}} \frac{\partial^{2} \gamma_{s}}{\partial \theta^{2}}=0, \text { in }\right] s, a[\times \mathcal{I} \\
\gamma_{\left.\right|_{\Gamma_{a}}}=0 \\
\left.\frac{\partial \gamma_{s}}{\partial \rho}\right|_{\Gamma_{s}}=h \\
\gamma_{s} 2 \pi-\text { periodic with respect to } \theta
\end{array}\right.
$$

and $r(s)=\beta_{s_{\Gamma_{s}}}$, where $\beta_{s}$ is the solution of

$$
\left\{\begin{array}{l}
\left.-\frac{1}{\rho} \frac{\partial}{\partial \rho}\left(\rho \frac{\partial \beta_{s}}{\partial \rho}\right)-\frac{1}{\rho^{2}} \frac{\partial^{2} \beta_{s}}{\partial \theta^{2}}=\hat{f}, \text { in }\right] s, a[\times \mathcal{I} \\
\beta_{s_{\Gamma}}=\hat{u}_{0} \\
\left.\frac{\partial \beta_{s}}{\partial \rho}\right|_{\Gamma_{s}}=0 \\
\beta_{s} 2 \pi-\text { periodic with respect to } \theta
\end{array}\right.
$$

By linearity of $\left(\hat{\mathcal{P}}_{s, h}\right)$ we have

$$
\left.\hat{u}_{s}\right|_{\Gamma_{s}}=P(s) h+r(s),
$$

where $P(s)$ is the Neumann to Dirichlet map for the annulus $\Omega \backslash \Omega_{s}$. 
Let $X=\left\{\hat{v} \mid \hat{v} \in L_{\rho}^{2}\left(0, a ; H_{\rho, P}^{1}(\mathcal{I})\right) \cap \frac{\partial \hat{u}}{\partial \rho} \in L_{\rho}^{2}\left(0, a ; L^{2}(\mathcal{I})\right)\right\}$, where $L_{\rho}^{2}$ stands for the space of functions of $\rho$ square integrable with the weight $\rho$. After passing to the limit, when $\varepsilon \rightarrow 0$, the factorization of problem $(\hat{\mathcal{P}})$ is synthesized by the following theorem

THEOREM 2 The solution $\hat{u}$ of $(\hat{\mathcal{P}})$ is the unique solution of the following system of uncoupled, first order in $\rho$, initial value problems

1. for every $h, \bar{h}$ in $L^{2}(\mathcal{I})$, the self-adjoint operator $P, P \leq 0$,

$$
\begin{aligned}
P \in L^{\infty}(0, a ; \quad & \mathcal{L}\left(L^{2}(\mathcal{I}), H_{\rho, P}^{1}(\mathcal{I})\right) \cap \mathcal{L}\left(H_{\rho, P}^{1 / 2}(\mathcal{I})^{\prime}, H_{\rho, P}^{1 / 2}(\mathcal{I})\right) \\
& \left.\cap \mathcal{L}\left(H_{\rho, P}^{1}(\mathcal{I})^{\prime}, L^{2}(\mathcal{I})\right)\right),
\end{aligned}
$$

satisfies the Riccati equation

$$
\left(\frac{\partial P}{\partial \rho} h, \bar{h}\right)+\left(\frac{1}{\rho^{2}} \frac{\partial}{\partial \theta} P h, \frac{\partial}{\partial \theta} P \bar{h}\right)-\left(\frac{1}{\rho} h, P \bar{h}\right)=(h, \bar{h})
$$

in $\mathcal{D}^{\prime}(0, a)$, with the initial condition $P(a)=0$;

2. for every $h$ in $L^{2}(\mathcal{I}), r \in X$ satisfies the equation

$$
\left(\frac{\partial r}{\partial \rho}, h\right)+\left(\frac{1}{\rho^{2}} \frac{\partial r}{\partial \theta}, \frac{\partial}{\partial \theta} P h\right)=(\hat{f}, P h)
$$

in $\mathcal{D}^{\prime}(0, a)$, with the initial condition $r(a)=\hat{u}_{0}$;

3. for every $h$ in $H_{\rho, P}^{1}(\mathcal{I})^{\prime}, \hat{u} \in X$ satisfies the equation

$$
-\left(\frac{\partial \hat{u}}{\partial \rho}, P h\right)+\langle\hat{u}, h\rangle_{H_{\rho, P}^{1}(\mathcal{I}), H_{\rho, P}^{1}(\mathcal{I})^{\prime}}=\langle r, h\rangle_{H_{\rho, P}^{1}(\mathcal{I}), H_{\rho, P}^{1}(\mathcal{I})^{\prime}}
$$

in $\mathcal{D}^{\prime}(0, a)$, with the initial condition $\hat{u}(0)=\lim _{\rho \rightarrow 0} r(\rho)$ in $L^{2}(\mathcal{I})$ which is constant.

$P, r$ and $\hat{u}$ thus defined are unique. Equations (4) and (5) are well posed for $\rho$ decreasing from a to 0 and $(6)$ is well posed for $\rho$ increasing from 0 to $a$.

The formal analogy between this result and the $L U$ Gauss factorization of a matrix should be emphasized. The Riccati equation (4) for $P$ is the analogous of the block $L U$ factorization of a block tridiagonal matrix, and the initial value problems (5) and (6) are the analogous of the lower and upper block triangular systems. This factorization inherits the well known property of the Gauss factorization for multiple right hand sides: if $(\mathcal{P})$ has to be solved for different $f$ and $u_{0},(4)$ is to be 
solved only once, then the initial value problems (5) and (6) are solved for each value of the data. Furthermore, if one considers a finite difference discretization of $(\mathcal{P})$ in polar coordinates, for example, one can show that the Gauss factorization of the obtained linear system can be obtained by one particular discretization of (4), (5), (6). But other possible discretizations exist with their own interest. Also this equivalent formulation of problem $(\mathcal{P})$ furnishes the Neumann to Dirichlet operator $P(s)$ for the annulus $\Omega \backslash \Omega_{s}$ which is of interest for various kinds of problems as domain decomposition or the definition of transparent boundary conditions.

\section{Sketch of the Proof of Theorem 2}

From (3), the solution $\hat{u}_{\varepsilon}$ of $\left(\hat{\mathcal{P}}_{\varepsilon}\right)$ in polar coordinates, satisfies the relation $\hat{u}_{\varepsilon}(\rho)=\left.P(\rho) \frac{\partial \hat{u}_{\varepsilon}}{\partial \rho}\right|_{\Gamma_{\rho}}+r(\rho), \forall \rho \in[\varepsilon, a]$. From this last equality, taking the derivative, in a formal way, with respect to $\rho$ and considering $\frac{\partial \hat{u}_{\epsilon}}{\partial \rho}$ arbitrary, we obtain $\frac{\partial P}{\partial \rho}-P \frac{1}{\rho^{2}} \frac{\partial^{2}}{\partial \theta^{2}} P-P \frac{1}{\rho}=I$ and $-P \hat{f}-P \frac{1}{\rho^{2}} \frac{\partial^{2} r}{\partial \theta^{2}}+$ $\frac{\partial r}{\partial \rho}=0$, and considering the boundary condition on $\Gamma_{a}$ in $\left(\mathcal{P}_{\varepsilon}\right)$, we obtain $P(a)=0$ and $r(a)=\hat{u}_{0}$.

From the two equations above, and respective initial conditions, we can obtain $P$ and $r$. Let $M$ and $N$ be defined by $M=\left\{v \in\left(H_{\rho, P}^{1 / 2}(\mathcal{I})\right)^{\prime} \mid\right.$ $\left.\int_{0}^{2 \pi} v \mathrm{~d} \theta=0\right\}$ and $N=M^{\perp}=\left\{v \in H_{\rho, P}^{1 / 2}(\mathcal{I}) \mid v\right.$ is constant $\}$. They are invariant by $P$. One has $L^{2}(\mathcal{I})=\left(M \cap L^{2}(\mathcal{I})\right) \oplus N$ and let $\Pi_{M}$ and $\Pi_{N}$ be the projection, for the $L^{2}(\mathcal{I})$ metrics, on each subspace respectively. The following theorem provides the initial condition for $\hat{u}_{\varepsilon}$ on $\Gamma_{\varepsilon}$.

THEOREM 3 Given $r(\varepsilon) \in L^{2}(\mathcal{I})$, there exists a unique solution $\hat{u}_{\varepsilon}(\varepsilon) \in$ $N$ and $\frac{\partial \hat{u}_{\varepsilon}}{\partial \rho}(\varepsilon) \in M$ for the equation $\hat{u}_{\varepsilon}(\varepsilon)=P(\varepsilon) \frac{\partial \hat{u}_{\varepsilon}}{\partial \rho}(\varepsilon)+r(\varepsilon)$. In particular, $\hat{u}_{\varepsilon}(\varepsilon)=\Pi_{N} r(\varepsilon)$.

We use the Galerkin method as in [5], [3], and adequate properties on the operator $P$ and function $r$. In finite dimension, we can prove the existence of a global solution of the decoupled system. Then we can justify the preceding formal calculation and we obtain, after passing to the limit when the dimension tends to infinity, the following result: 
THEOREM 4 For every $h, \bar{h}$ in $L^{2}(\mathcal{I})$, the operator $P$ belongs to $L^{\infty}\left(\varepsilon, a ; \mathcal{L}\left(H_{\rho, P}^{1 / 2}(\mathcal{I})^{\prime}, H_{\rho, P}^{1 / 2}(\mathcal{I})\right)\right)$ and satisfies the following equation

$$
\left(\frac{\partial P}{\partial \rho} h, \bar{h}\right)+\left(\frac{1}{\rho^{2}} \frac{\partial}{\partial \theta} P h, \frac{\partial}{\partial \theta} P \bar{h}\right)-\left(\frac{1}{\rho} h, P \bar{h}\right)=(h, \bar{h}),
$$

in $\mathcal{D}^{\prime}(\varepsilon, a)$, with $P(a)=0$. The function $r$ belongs to $\left.X\right|_{\Omega \backslash \Omega_{\varepsilon}}$, satisfies $r(a)=\hat{u}_{0}$ and for every $h$ in $L^{2}(\mathcal{I})$, satisfies in $\mathcal{D}^{\prime}(\varepsilon, a)$ the following equation

$$
\left(\frac{\partial r}{\partial \rho}, h\right)+\left(\frac{1}{\rho^{2}} \frac{\partial r}{\partial \theta}, \frac{\partial}{\partial \theta} P h\right)=(\hat{f}, P h) .
$$

Since $P$ and $r$ do not depend on $\varepsilon$ and thanks to the estimates on $P(\rho)$ and $r(\rho)$, we can take $\varepsilon$ arbitrarily small and consequently consider the previous equalities defined on $\mathcal{D}^{\prime}(0, a)$. Let $\|\cdot\|_{\rho}$ denote the norm in $\mathcal{L}\left(H_{\rho, P}^{1 / 2}(\mathcal{I})^{\prime}, H_{\rho, P}^{1 / 2}(\mathcal{I})\right)$. The following theorem gives the behavior of $P$ and $r$ around the origin which provides the regularity claimed in Theorem 2:

Theorem 5 For $P$ satisfying $(7)$ and $P(a)=0$, we have $\lim _{\rho \rightarrow 0}\|P(\rho)\|_{\rho}=$ 1. Furthermore $\lim _{\rho \rightarrow 0}\left\|P(\rho)-\rho\left(P_{\infty} o \Pi_{M}\right)\right\|_{\rho}=0$, where $P_{\infty}$ is the negative self-adjoint operator satisfying $-P_{\infty} \frac{\partial^{2}}{\partial \theta^{2}} P_{\infty}=I$.

The solution $r$ of $(8)$ and $r(a)=\hat{u}_{0}$, has a limit $r(0)$ constant with respect to $\theta: \lim _{\rho \rightarrow 0}\|r(\rho)-r(0)\|_{L^{2}(\mathcal{I})}=0$.

It should be also remarked that, measured with the fixed norm $\|\cdot\|_{\mathcal{L}\left(L^{2}(\mathcal{I}), L^{2}(\mathcal{I})\right)}, P(\rho)$ goes to 0 as $\rho$ goes to 0 . Concerning the equation on $\hat{u}_{\varepsilon}$ we have

THEOREM 6 For every $h$ in $H_{\rho, P}^{1}(\mathcal{I})^{\prime}, \hat{u}_{\varepsilon}$ satisfies in $\mathcal{D}^{\prime}(\varepsilon, a)$ the following equation

$$
-\left(\frac{\partial \hat{u}_{\varepsilon}}{\partial \rho}, P h\right)+\left\langle\hat{u}_{\varepsilon}, h\right\rangle_{H_{\rho, P}^{1}(\mathcal{I}), H_{\rho, P}^{1}(\mathcal{I})^{\prime}}=\langle r, h\rangle_{H_{\rho, P}^{1}(\mathcal{I}), H_{\rho, P}^{1}(\mathcal{I})^{\prime}},
$$

with the initial condition $\hat{u}_{\varepsilon}(\varepsilon)=\Pi_{N} r(\varepsilon)$.

Using Theorem 1 we obtain the convergence in $X$ of $\hat{u}_{\varepsilon}$ to $\hat{u}$ satisfying (6). Furthermore, thanks to the local regularity assumption on $f$ near the origin which implies that $u \in \mathcal{C}^{2, \alpha}(\mathcal{O})$, one can prove that $\lim _{\varepsilon \rightarrow 0} \hat{u}_{\varepsilon}(\varepsilon)=\hat{u}(0)$ (the proof for the uniqueness of the solution of $(6)$ uses the determination of $\hat{u}(0))$. 


\section{Factorization by Invariant Embedding: Dual Case}

Another factorization could be obtained by using an invariant embedding defined by the family of disks $\Omega_{s}$. Here the main difficulty is to define the initial conditions for $P$ and $r$ at the origin.

We embed problem $\left(\mathcal{P}_{\varepsilon}\right)$ in a family of similar problems $\left(\tilde{\mathcal{P}}_{\varepsilon, h}\right)$ defined on the annulus $\left.\Omega_{s} \backslash \Omega_{\varepsilon}, s \in\right] \varepsilon, a$ [ and satisfying an additional Robin boundary condition in $\Gamma_{s}$. We find the following problem in polar coordinates:

$$
\left(\hat{\tilde{\mathcal{P}}}_{\varepsilon, h}\right)\left\{\begin{array}{l}
\left.-\frac{1}{\rho} \frac{\partial}{\partial \rho}\left(\rho \frac{\partial \hat{u}_{\varepsilon}}{\partial \rho}\right)-\frac{1}{\rho^{2}} \frac{\partial^{2} \hat{u}_{\varepsilon}}{\partial \theta^{2}}=\hat{f}, \text { in }\right] \varepsilon, s[\times \mathcal{I} \\
\hat{u}_{\varepsilon} \mid \Gamma_{\varepsilon} \text { constant, } \hat{u}_{\varepsilon} \quad 2 \pi-\text { periodic with respect to } \theta \\
\left.\int_{0}^{2 \pi} \frac{\partial \hat{u}_{\varepsilon}}{\partial \rho}\right|_{\Gamma_{\varepsilon}} \mathrm{d} \theta=0 \\
\left.\frac{\partial \hat{u}_{\varepsilon}}{\partial \rho}\right|_{\Gamma_{s}}+\alpha \hat{u}_{\varepsilon_{\Gamma_{s}}}=h, \quad \alpha>0
\end{array}\right.
$$

It is clear that $\left(\hat{\mathcal{P}}_{\varepsilon}\right)$ is exactly $\left(\hat{\tilde{\mathcal{P}}}_{\varepsilon, h}\right)$ for $s=a$ and $h=\left.\frac{\partial \hat{u}_{\varepsilon}}{\partial \rho}\right|_{\Gamma_{s}}+\alpha \hat{u}_{0}$, and so we use the same notation $\hat{u}_{\varepsilon}$ for the solution of $\left(\hat{\mathcal{P}}_{\varepsilon}\right)$ and the family of solutions of $\left(\hat{\tilde{\mathcal{P}}}_{\varepsilon, h}\right)$. This should not make confusion with the solutions of $\left(\hat{\mathcal{P}}_{s, h}\right)$ in the previous section. For every $s \in(\varepsilon, a]$ and $h \in H_{\rho, P}^{1 / 2}(\mathcal{I})^{\prime}$ we define $\tilde{P}_{\varepsilon}(s) h=\left.\tilde{\gamma}_{\varepsilon}\right|_{\Gamma_{s}}$, where $\tilde{\gamma}_{\varepsilon}$ is the solution of

$$
\left\{\begin{array}{l}
\left.-\frac{1}{\rho} \frac{\partial}{\partial \rho}\left(\rho \frac{\partial \tilde{\gamma}_{\varepsilon}}{\partial \rho}\right)-\frac{1}{\rho^{2}} \frac{\partial^{2} \tilde{\gamma}_{\varepsilon}}{\partial \theta^{2}}=0, \text { in }\right] \varepsilon, s[\times \mathcal{I} \\
\tilde{\gamma}_{\varepsilon_{\left.\right|_{\varepsilon}}} \text { constant, } \tilde{\gamma}_{\varepsilon} 2 \pi-\text { periodic with respect to } \theta \\
\left.\int_{0}^{2 \pi} \frac{\partial \tilde{\gamma}_{\varepsilon}}{\partial \rho}\right|_{\Gamma_{\varepsilon}} \mathrm{d} \theta=0 \\
\left.\frac{\partial \tilde{\gamma}_{\varepsilon}}{\partial \rho}\right|_{\Gamma_{s}}+\left.\alpha \tilde{\gamma}_{\varepsilon}\right|_{\Gamma_{s}}=h
\end{array}\right.
$$

and $\tilde{r}_{\varepsilon}(s)=\tilde{\beta}_{\varepsilon_{\Gamma_{s}}}$, where $\tilde{\beta}_{\varepsilon}$ is the solution of

$$
\left\{\begin{array}{l}
\left.-\frac{1}{\rho} \frac{\partial}{\partial \rho}\left(\rho \frac{\partial \tilde{\beta}_{\varepsilon}}{\partial \rho}\right)-\frac{1}{\rho^{2}} \frac{\partial^{2} \tilde{\beta}_{\varepsilon}}{\partial \theta^{2}}=\hat{f}, \text { in }\right] \varepsilon, s[\times \mathcal{I} \\
\tilde{\beta}_{\varepsilon_{\mid \Gamma_{\varepsilon}}} \text { constant, } \tilde{\beta}_{\varepsilon} \quad 2 \pi \text { - periodic with respect to } \theta \\
\left.\int_{0_{\tilde{R}_{\varepsilon}}^{2 \pi}}^{2 \pi} \frac{\partial \tilde{\beta}_{\varepsilon}}{\partial \rho}\right|_{\Gamma_{\varepsilon}} \mathrm{d} \theta=0 \\
\left.\frac{\partial \tilde{\beta}_{\varepsilon}}{\partial \rho}\right|_{\Gamma_{s}}+\alpha \tilde{\beta}_{\varepsilon} \mid \Gamma_{s}=0
\end{array}\right.
$$


By linearity of $\left(\tilde{\mathcal{P}}_{\varepsilon, h}\right)$ we have

$$
\hat{u}_{\varepsilon_{\mid \Gamma_{s}}}=\tilde{P}_{\varepsilon}(s)\left(\left.\frac{\partial \hat{u}_{\varepsilon}}{\partial \rho}\right|_{\Gamma_{s}}+\left.\alpha \hat{u}_{\varepsilon}\right|_{\Gamma_{s}}\right)+\tilde{r}_{\varepsilon}(s), \forall s \in[\varepsilon, a] .
$$

The following theorem gives another factorization of problem $(\hat{\mathcal{P}})$ :

THEOREM 7 The solution $\hat{u}$ of $(\hat{\mathcal{P}})$ is the unique solution of the following system of uncoupled, first order in $\rho$, initial value problem:

1. for every $h, \bar{h}$ in $L^{2}(\mathcal{I})$, the self-adjoint operator $\tilde{P}, \tilde{P} \geq 0$,

$$
\begin{aligned}
\tilde{P} \in L^{\infty}(0, a ; & \mathcal{L}\left(L^{2}(\mathcal{I}), H_{\rho, P}^{1}(\mathcal{I})\right) \cap \mathcal{L}\left(H_{\rho, P}^{1 / 2}(\mathcal{I})^{\prime}, H_{\rho, P}^{1 / 2}(\mathcal{I})\right) \\
& \left.\cap \mathcal{L}\left(H_{\rho, P}^{1}(\mathcal{I})^{\prime}, L^{2}(\mathcal{I})\right)\right),
\end{aligned}
$$

satisfies the Riccati equation

$$
\begin{aligned}
& \left(\frac{\partial \tilde{P}}{\partial \rho} h, \bar{h}\right)-\left(\frac{1}{\rho} h, \tilde{P} \bar{h}\right)+\frac{1}{\rho} \alpha(\tilde{P} h, \tilde{P} \bar{h})+2 \alpha(\tilde{P} h, \bar{h}) \\
& +\left(\frac{1}{\rho^{2}} \frac{\partial}{\partial \theta} \tilde{P} h, \frac{\partial}{\partial \theta} \tilde{P} \bar{h}\right)-\alpha^{2}(\tilde{P} h, \tilde{P} \bar{h})=(h, \bar{h})
\end{aligned}
$$

in $\mathcal{D}^{\prime}(0, a)$, with the initial condition $\tilde{P}(0)=\frac{1}{\alpha} \Pi_{N}$;

2. for every $h$ in $L^{2}(\mathcal{I}), \tilde{r} \in X$ satisfies the equation

$$
\begin{aligned}
& \left(\frac{1}{\rho} \alpha \tilde{r}, \tilde{P} h\right)+\left(\frac{1}{\rho^{2}} \frac{\partial \tilde{r}}{\partial \theta}, \frac{1}{\partial \theta} \tilde{P} h\right)-\alpha^{2}(\tilde{r}, \tilde{P} h)+\left(\frac{\partial \tilde{r}}{\partial \rho}, h\right) \\
& +\alpha(\tilde{r}, h)=(f, \tilde{P} h)
\end{aligned}
$$

in $\mathcal{D}^{\prime}(0, a)$, with the initial condition $\tilde{r}(0)=0$;

3. for every $h$ in $H_{\rho, P}^{1}(\mathcal{I})^{\prime}, \hat{u} \in X$ satisfies the equation

$$
\begin{aligned}
& -\left(\frac{\partial \hat{u}}{\partial \rho}+\alpha \hat{u}, \tilde{P} h\right)+\langle\hat{u}, h\rangle_{H_{\rho, P}^{1}(\mathcal{I}), H_{\rho, P}^{1}(\mathcal{I})^{\prime}} \\
& =\langle\tilde{r}, h\rangle_{H_{\rho, P}^{1}(\mathcal{I}), H_{\rho, P}^{1}(\mathcal{I})^{\prime}}
\end{aligned}
$$

in $\mathcal{D}^{\prime}(0, a)$, with the initial condition $\hat{u}(a)=\hat{u}_{0}$.

Equations (11) and (12) are well posed for $\rho$ increasing from 0 to $a$ and (13) is well posed for $\rho$ decreasing from a to $0 . \tilde{P}, \tilde{r}$ and $\hat{u}$ thus defined are unique. 


\section{Sketch of the Proof of Theorem 7}

From $(10)$, the solution $\hat{u}_{\varepsilon}$ of $\left(\tilde{\mathcal{P}}_{\varepsilon}\right)$ satisfies

$$
\hat{u}_{\varepsilon}(\rho)=\tilde{P}_{\varepsilon}(\rho)\left(\left.\frac{\partial \hat{u}_{\varepsilon}}{\partial \rho}\right|_{\Gamma_{\rho}}+\alpha \hat{u}_{\varepsilon_{\mid} \rho}\right)+\tilde{r}_{\varepsilon}(\rho), \forall \rho \in[\varepsilon, a] .
$$

Taking the derivative in a formal way with respect to $\rho$ and considering $\frac{\partial \hat{u}_{\varepsilon}}{\partial \rho}+\alpha \hat{u}_{\varepsilon}$ arbitrary, we obtain the following system

$$
\left\{\begin{array}{l}
\frac{\partial \tilde{P}_{\varepsilon}}{\partial \rho}-\frac{\tilde{P}_{\varepsilon}}{\rho}+\frac{1}{\rho} \alpha \tilde{P}_{\varepsilon}^{2}-\frac{1}{\rho^{2}} \tilde{P}_{\varepsilon} \frac{\partial^{2}}{\partial \theta^{2}} \tilde{P}_{\varepsilon}+2 \alpha \tilde{P}_{\varepsilon}-\left(\alpha \tilde{P}_{\varepsilon}\right)^{2}=I \\
-\tilde{P}_{\varepsilon} f+\frac{1}{\rho} \tilde{P}_{\varepsilon} \alpha \tilde{r}_{\varepsilon}-\frac{1}{\rho^{2}} \tilde{P}_{\varepsilon} \frac{\partial^{2} \tilde{r}_{\varepsilon}}{\partial \theta^{2}}-\alpha^{2} \tilde{P}_{\varepsilon} \tilde{r}_{\varepsilon}+\frac{\partial \tilde{r}_{\varepsilon}}{\partial \rho}+\alpha \tilde{r}_{\varepsilon}=0 \\
\hat{u}_{\varepsilon}=\tilde{P}_{\varepsilon}\left(\frac{\partial \hat{u}_{\varepsilon}}{\partial \rho}+\alpha \hat{u}_{\varepsilon}\right)+\tilde{r}_{\varepsilon}
\end{array}\right.
$$

We have $\hat{u}_{\varepsilon}(a)=\hat{u}_{0}$. Further, considering the sets $M=\left\{v \in\left(H_{\tau, P}^{1 / 2}(\mathcal{I})\right)^{\prime} \mid\right.$ $\left.\int_{0}^{2 \pi} v \mathrm{~d} \theta=0\right\}, N=M^{\perp}=\left\{v \in H_{\tau, P}^{1 / 2}(\mathcal{I}) \mid v\right.$ is constant $\}$ and the operators $\Pi_{M}, \Pi_{N}$ as previously, one has $\tilde{P}_{\varepsilon}: M \rightarrow M, \tilde{P}_{\varepsilon}: N \rightarrow N$ and from (10) we obtain

$$
\begin{array}{cc} 
& \Pi_{M} \hat{u}_{\varepsilon}(\varepsilon)=\tilde{P}_{\varepsilon}(\varepsilon)\left(\Pi_{M} \frac{\partial \hat{u}_{\varepsilon}}{\partial \rho}(\varepsilon)+\alpha \Pi_{M} \hat{u}_{\varepsilon}(\varepsilon)\right)+\Pi_{M} \tilde{r}_{\varepsilon}(\varepsilon) \\
\Rightarrow \quad & 0=\tilde{P}_{\varepsilon}(\varepsilon) \Pi_{M} \frac{\partial \hat{u}_{\varepsilon}}{\partial \rho}(\varepsilon)+\Pi_{M} \tilde{r}_{\varepsilon}(\varepsilon) \Rightarrow \Pi_{M} \tilde{P}_{\varepsilon}(\varepsilon)=0, \Pi_{M} \tilde{r}_{\varepsilon}(\varepsilon)=0 \\
& \Pi_{N} \hat{u}_{\varepsilon}(\varepsilon)=\tilde{P}_{\varepsilon}(\varepsilon)\left(\Pi_{N} \frac{\partial \hat{u}_{\varepsilon}}{\partial \rho}(\varepsilon)+\alpha \Pi_{N} \hat{u}_{\varepsilon}(\varepsilon)\right)+\Pi_{N} \tilde{r}_{\varepsilon}(\varepsilon) \\
\Rightarrow & \hat{u}_{\varepsilon}(\varepsilon)=\alpha \tilde{P}_{\varepsilon}(\varepsilon) \hat{u}_{\varepsilon}(\varepsilon)+\Pi_{N} \tilde{r}_{\varepsilon}(\varepsilon) \Rightarrow \Pi_{N} \tilde{P}_{\varepsilon}(\varepsilon)=\frac{I}{\alpha}, \Pi_{N} \tilde{r}_{\varepsilon}(\varepsilon)=0
\end{array}
$$

From (14) and (15) we obtain $\tilde{r}_{\varepsilon}(\varepsilon)=\Pi_{M} \tilde{r}_{\varepsilon}(\varepsilon)+\Pi_{N} \tilde{r}_{\varepsilon}(\varepsilon)=0$. In the same way, since $\tilde{P}_{\varepsilon}(\varepsilon) h=\tilde{P}_{\varepsilon}(\varepsilon) \Pi_{M} h+\tilde{P}_{\varepsilon}(\varepsilon) \Pi_{N} h=\frac{1}{\alpha} \Pi_{N} h$ we obtain $\tilde{P}_{\varepsilon}(\varepsilon)=\frac{1}{\alpha} \Pi_{N}$.

Using again the Galerkin method, we can justify these formal calculations through the adequate proprieties on $\tilde{P}_{\varepsilon}$ and $\tilde{r}_{\varepsilon}$. After passing to the limit when the dimension tends to infinity, we find the following result, by the same reasoning as in section 4 : 
THEOREM 8 1. For every $h, \bar{h}$ in $L^{2}(\mathcal{I})$, the operator $\tilde{P}_{\varepsilon}$ belongs to $L^{\infty}\left(\varepsilon, a ; \mathcal{L}\left(H_{\rho, P}^{1 / 2}(\mathcal{I})^{\prime}, H_{\rho, P}^{1 / 2}(\mathcal{I})\right)\right)$ and satisfies the following equation

$$
\begin{aligned}
& \left(\frac{\partial \tilde{P}_{\varepsilon}}{\partial \rho} h, \bar{h}\right)-\left(\frac{1}{\rho} h, \tilde{P}_{\varepsilon} \bar{h}\right)+\left(\frac{1}{\rho^{2}} \frac{\partial}{\partial \theta} \tilde{P}_{\varepsilon} h, \frac{\partial}{\partial \theta} \tilde{P}_{\varepsilon} \bar{h}\right) \\
& +\frac{1}{\rho} \alpha\left(\tilde{P}_{\varepsilon} h, \tilde{P}_{\varepsilon} \bar{h}\right)+2 \alpha\left(\tilde{P}_{\varepsilon} h, \bar{h}\right)-\alpha^{2}\left(\tilde{P}_{\varepsilon} h, \tilde{P}_{\varepsilon} \bar{h}\right)=(h, \bar{h}),
\end{aligned}
$$

in $\mathcal{D}^{\prime}(\varepsilon, a)$, considering $\tilde{P}_{\varepsilon}(\varepsilon)=\frac{1}{\alpha} \Pi_{N}$.

2. The function $\tilde{r}_{\varepsilon}$ belongs to $X_{\left.\right|_{\Omega_{s} \backslash \Omega_{\varepsilon}}}$, satisfies $\tilde{r}_{\varepsilon}(\varepsilon)=0$, and for every $h$ in $L^{2}(\mathcal{I})$ verifies, in $\mathcal{D}^{\prime}(\varepsilon, a)$, the following equation

$$
\begin{aligned}
& \left(\frac{1}{\rho} \alpha \tilde{r}_{\varepsilon}, \tilde{P}_{\varepsilon} h\right)+\left(\frac{1}{\rho^{2}} \frac{\partial \tilde{r}_{\varepsilon}}{\partial \theta}, \frac{1}{\partial \theta} \tilde{P}_{\varepsilon} h\right)-\alpha^{2}\left(\tilde{r}_{\varepsilon}, \tilde{P}_{\varepsilon} h\right)+\left(\frac{\partial \tilde{r}_{\varepsilon}}{\partial \rho}, h\right) \\
& +\alpha\left(\tilde{r}_{\varepsilon}, h\right)=\left(f, \tilde{P}_{\varepsilon} h\right) .
\end{aligned}
$$

3. For every $h$ in $H_{\rho, P}^{1}(\mathcal{I})^{\prime}, \hat{u}_{\varepsilon}$ satisfies the following equation

$$
\begin{aligned}
& -\left(\frac{\partial \hat{u}_{\varepsilon}}{\partial \rho}+\alpha \hat{u}_{\varepsilon}, \tilde{P}_{\varepsilon} h\right)+\left\langle\hat{u}_{\varepsilon}, h\right\rangle_{H_{\rho, P}^{1}(\mathcal{I}), H_{\rho, P}^{1}(\mathcal{I})^{\prime}} \\
& =\left\langle\tilde{r}_{\varepsilon}, h\right\rangle_{H_{\rho, P}^{1}(\mathcal{I}), H_{\rho, P}^{1}(\mathcal{I})^{\prime}}
\end{aligned}
$$

in $\mathcal{D}^{\prime}(\varepsilon, a)$, with $\hat{u}_{\varepsilon}(a)=\hat{u}_{0}$.

Using Theorem 1 we obtain the convergence in $X$ of $\hat{u}_{\varepsilon}$ to $\hat{u}$ satisfying (13). Now, since $\tilde{P}_{\varepsilon}$ and $\tilde{r}_{\varepsilon}$ depend on $\varepsilon$ and considering $\hat{u}(\rho)=\tilde{P}(\rho) h+$ $\tilde{r}(\rho)$ we use the following consequence of Theorem 1 :

Corollary 9 For all $\rho \in[0, a], \tilde{r}_{\varepsilon}(\rho) \rightarrow \tilde{r}(\rho)$ strongly in $H_{\rho, P}^{1 / 2}(\mathcal{I})$, when $\varepsilon \rightarrow 0$. Also, for all $\rho \in[0, a]$ and for a fixed $h, \tilde{P}_{\varepsilon}(\rho) h \rightarrow \tilde{P}(\rho) h$, strongly in $H_{\rho, P}^{1 / 2}(\mathcal{I})$ and weakly in $H_{\rho, P}^{3 / 2}(\mathcal{I})$, when $\varepsilon \rightarrow 0$.

Therefore, passing to the limit when $\varepsilon \rightarrow 0$ we find $\tilde{P}$ and $\tilde{r}$ satisfying (11) and (12), respectively.

Using again the appropriate conditions of regularity around the origin (that is, $f \in \mathcal{C}^{0, \alpha}(\Omega)$ ), we can define the value of $\hat{u}(0)$ (as a constant), and consequently we have $\hat{u}(0) \in N$. Also, since $\frac{\partial \hat{u}}{\partial \rho}=\frac{\partial u}{\partial x} \cos (\theta)+\frac{\partial u}{\partial y} \sin (\theta)$ 
and we have assumed enough regularity around the origin, we have $\int_{0}^{2 \pi} \frac{\partial \hat{u}}{\partial \rho}(0) \mathrm{d} \theta=\int_{0}^{2 \pi} c_{1} \cos (\theta)+c_{2} \sin (\theta) \mathrm{d} \theta=0$, from which we conclude that $\frac{\partial \hat{u}}{\partial \rho}(0) \in M$. Therefore, from $\hat{u}_{\left.\right|_{\Gamma_{s}}}=\tilde{P}(s)\left(\left.\frac{\partial \hat{u}}{\partial \rho}\right|_{\Gamma_{s}}+\alpha \hat{u}_{\Gamma_{s}}\right)+$ $\tilde{r}(s), \forall s \in[0, a]$ we obtain

$$
\begin{gathered}
\Pi_{M} \hat{u}(0)=\tilde{P}(0)\left(\Pi_{M} \frac{\partial \hat{u}}{\partial \rho}(0)+\alpha \Pi_{M} \hat{u}(0)\right)+\Pi_{M} \tilde{r}(0) \\
\Rightarrow \quad 0=\tilde{P}(0) \Pi_{M} \frac{\partial \hat{u}}{\partial \rho}(0)+\Pi_{M} \tilde{r}(0) \Rightarrow \Pi_{M} \tilde{P}(0)=0, \Pi_{M} \tilde{r}(0)=0, \\
\Pi_{N} \hat{u}(0)=\tilde{P}(0)\left(\Pi_{N} \frac{\partial \hat{u}}{\partial \rho}(0)+\alpha \Pi_{N} \hat{u}(0)\right)+\Pi_{N} \tilde{r}(0) \\
\Rightarrow \quad \hat{u}(0)=\alpha \tilde{P}(0) \hat{u}(0)+\Pi_{N} \tilde{r}(0) \Rightarrow \Pi_{N} \tilde{P}(0)=\frac{1}{\alpha} I, \Pi_{N} \tilde{r}(0)=0 .
\end{gathered}
$$

From (16) and (17) we obtain $\tilde{r}(0)=\Pi_{M} \tilde{r}(0)+\Pi_{N} \tilde{r}(0)=0$. In the same way, since $\tilde{P}(0) h=\tilde{P}(0) \Pi_{M} h+\tilde{P}(0) \Pi_{N} h=\frac{1}{\alpha} \Pi_{N} h$ we obtain $\tilde{P}(0)=\frac{1}{\alpha} \Pi_{N}$.

\section{Final Remarks}

We believe that this method is much more general than presented here and that it can be extended to higher dimensions, to other operators than the Laplacian and more general domains. For example for star shaped domains the embedding can be done by homothety, taking the angle $\theta$ and the homothety factor as independent variables. Then the singularity at the origin is treated in the same way.

\section{References}

[1] E. Angel and R. Bellman. Dynamic Programming and Partial Differential Equations. Academic Press, London, 1972.

[2] R. Bellman. Dynamic Programming. Princeton University Press, Princeton, 1957.

[3] J. Henry and A.M. Ramos. Factorization of second order elliptic boundary value problems by dynamic programming. to appear in Nonlinear Analysis, T.M.A..

[4] J. Henry, B. Louro and M.C. Soares. A factorization method for elliptic problems in a circular domain. to appear in CRAS.

[5] J.-L. Lions. Contrôle optimal de systèmes gouvernés par des équations aux dérivées partielles. Dunod, Paris, 1968. 